Bundesgesundheitsbl 2014 $57: 13-21$ DOI 10.1007/s00103-013-1878-z

Online publiziert: 20. Dezember 2013

c) Springer-Verlag Berlin Heidelberg 2013
K. Kraywinkel ${ }^{1}$ - B. Barnes ${ }^{1} \cdot$ S. Dahm ${ }^{1} \cdot$ J. Haberland ${ }^{1} \cdot$ A. Nennecke ${ }^{2} \cdot$ R. Stabenow ${ }^{3}$

${ }^{1}$ Zentrum für Krebsregisterdaten, Robert Koch-Institut, Berlin

${ }^{2}$ Hamburgisches Krebsregister, Hamburg

${ }^{3}$ Gemeinsames Krebsregister der neuen Bundesländer und Berlins, Berlin

\title{
Von regionalen Daten zu bundesweiten Aussagen
}

\author{
Die Methoden im Zentrum \\ für Krebsregisterdaten
}

\section{Einleitung}

Im Bundeskrebsregisterdatengesetz (BKRG) von 2009 werden die Bundesländer erstmals verpflichtet, Daten $\mathrm{zu}$ Krebsneuerkrankungen flächendeckend und vollzählig zu erheben und an das Zentrum für Krebsregisterdaten (ZfKD) am Robert Koch-Institut (RKI) zu übermitteln [1]. Seit dem gleichen Jahr besteht mit dem Inkrafttreten des Krebsregistergesetzes Baden-Württemberg in allen Bundesländern auch die gesetzliche Grundlage für eine flächendeckende Krebsregistrierung. Das ZfKD ist nach BKRG unter anderem damit beauftragt, die Vollzähligkeit der Erfassung in den Registern zu überprüfen und bundesweite Inzidenz- und Überlebensraten zu schätzen. Diese Aufgaben wurden auch schon vor 2009 von der Dachdokumentation Krebs am RKI wahrgenommen [2, 3].

Die vollzählige Erfassung von Krebsneuerkrankungen kann auch bei bestehenden gesetzlichen Grundlagen und der inzwischen in fast allen Ländern bestehenden Meldepflicht nicht a priori angenommen werden, sondern ist ein dynamischer, von vielen Faktoren beeinflusster Prozess. Einige Register befinden sich noch in der Aufbauphase, in anderen ist etwa die Weiterleitung von Erkrankungsmeldungen bei einer Behandlung außerhalb des zuständigen Wohnortregisters oder die Erfassung von Wegzügen von an Krebs erkrankten Personen aus dem Re- gistergebiet noch nicht befriedigend geregelt. Unabhängig davon spiegeln sich unterschiedliche gesetzliche, strukturelle und finanzielle Rahmenbedingungen in den Ländern teilweise in einer unterschiedlichen Qualität der Erfassung von Erkrankungs- und Sterbefällen wider. Schließlich ist es auch aufgrund stetig gewachsener Dokumentationsverpflichtungen in der onkologischen Versorgung eine besondere Herausforderung für die Register, die Motivation der Melder für die Krebsregistrierung aufzubauen und aufrechtzuerhalten. Die Meldepflicht alleine bietet nach allen Erfahrungen hierfür keine Garantie, zumal die Register meist nur wenig Handhabe haben, diese auch durchzusetzen.

Soweit Krebsneuerkrankungen bzw. Sterbefälle von an Krebs erkrankten Personen in Deutschland nicht vollzählig erfasst werden, müssen demnach bundesweite Aussagen zu Inzidenz, Überleben und Prävalenz von Krebserkrankungen vorerst noch über mehr oder weniger komplexe Schätzverfahren gewonnen werden. Da die Ergebnisse sowohl möglichst repräsentativ als auch wenig verzerrt sein sollen, beruhen die Schätzungen des ZfKD auf den Daten derjenigen Register, die als ausreichend vollzählig bewertet werden. Bereits seit den 1990er Jahren ging die Dachdokumentation Krebs nach diesem Prinzip vor $[2,3]$. Um der inzwischen breiteren Datenbasis und den mit dem BKRG gewachsenen
Anforderungen an die zentrale Auswertungsstelle gerecht $\mathrm{zu}$ werden, wurden die Methoden nach Gründung des ZfKD seit 2009 unter Beteiligung von Vertretern der Register und externen Wissenschaftlern überarbeitet. Der folgende Beitrag bietet einen Überblick über die aktuell vom ZfKD verwendeten Schätzund Berechnungsverfahren. Da die Vollzähligkeitsbewertung der Erfassung von Neuerkrankungen und Sterbefällen in den einzelnen Registern sowie die Schätzung bundesweiter Ergebnisse unmittelbar zusammenhängen, werden die angewandten Methoden im Folgenden unter den jeweiligen Oberbegriffen „Inzidenz“ und „Überleben“ gemeinsam dargestellt. Zusätzlich wird die Methode der Prävalenzschätzung kurz skizziert.

\section{Die verschiedenen \\ Schätzverfahren im Zentrum für Krebsregisterdaten}

\section{Inzidenz: Schätzung der Vollzähligkeit der Erfassung} von Neuerkrankungen

Das Zentrum für Krebsregisterdaten schätzt den Erfassungsgrad der bevölkerungsbezogenen Landeskrebsregister mithilfe des Verhältnisses von Mortalität zu Inzidenz (M/I-Index). Diesem Verfahren liegt die Annahme zugrunde, dass sich Diagnostik, Therapie und damit auch Überlebensaussichten für $\mathrm{Pa}$ - 


\begin{tabular}{|c|c|}
\hline ICD-10 & Lokalisationen \\
\hline $\begin{array}{l}\text { C00-C14, } \\
\text { C30-C32 }\end{array}$ & Kopf- und Halstumore \\
\hline $\mathrm{C} 15-\mathrm{C} 16$ & Speiseröhre und Magen \\
\hline $\begin{array}{l}\text { C17-C21, } \\
\text { C26 }\end{array}$ & Darm \\
\hline $\mathrm{C} 22-\mathrm{C} 25$ & $\begin{array}{l}\text { Leber, Galle und Bauchspeichel- } \\
\text { drüse }\end{array}$ \\
\hline $\begin{array}{l}\text { C33-C39, } \\
\text { C45 }\end{array}$ & Thoraxorgane \\
\hline $\begin{array}{l}\text { C40-C41, } \\
\text { C46-C49 }\end{array}$ & $\begin{array}{l}\text { Knochen, Knorpel und Weich- } \\
\text { teile }\end{array}$ \\
\hline C43 & Malignes Melanom \\
\hline C50 & Brustdrüse \\
\hline C51-C53 & Vulva, Vagina, Gebärmutterhals \\
\hline C54-C55 & Gebärmutterkörper \\
\hline C56-C58 & $\begin{array}{l}\text { Eierstöcke und sonstige weib- } \\
\text { liche Geschlechtsorgane }\end{array}$ \\
\hline C61 & Prostata \\
\hline $\begin{array}{l}\mathrm{C} 60, \mathrm{C} 62- \\
\mathrm{C} 63\end{array}$ & $\begin{array}{l}\text { Penis, Hoden und sonstige } \\
\text { männliche Geschlechtsorgane }\end{array}$ \\
\hline $\begin{array}{l}\text { C64-C68, } \\
\text { C74 }\end{array}$ & Harnorgane und Nebenniere \\
\hline C69-C72 & Zentrales Nervensystem \\
\hline $\mathrm{C} 73$ & Schilddrüse \\
\hline C81-C96 & $\begin{array}{l}\text { Blutbildendes und lymphati- } \\
\text { sches System }\end{array}$ \\
\hline $\begin{array}{l}\text { C75-C80, } \\
\text { C97 }\end{array}$ & $\begin{array}{l}\text { Ungenau bezeichnete/unbe- } \\
\text { kannte Lokalisationen }\end{array}$ \\
\hline
\end{tabular}

tienten gleichen Geschlechts, gleichen Alters und mit der gleichen Krebserkrankung innerhalb Deutschlands nicht wesentlich unterscheiden und sich regional unterschiedliche Krebsrisiken damit auch in der amtlichen Todesursachenstatistik abbilden. Unter dieser Voraussetzung lässt sich mithilfe des M/I-Index einer als vollzählig angenommenen Referenzregion unter Verwendung der regionalen Mortalität die zu erwartende Inzidenz in einer zu untersuchenden Region schätzen.

Die so gewonnenen Erwartungswerte werden anschließend mit den in der Untersuchungsregion tatsächlich erhobenen Daten verglichen. Der Quotient aus beobachteten und erwarteten Fällen, aufsummiert über alle Altersgruppen, ergibt den geschätzten Erfassungsgrad. Basis für die Schätzung sind hierbei nur die ärztlich gemeldeten Fälle. Erkrankungsfälle, die dem Register nur über eine Todesbescheinigung bekannt werden (Death
Certificate Only - DCO), werden hierbei nicht berücksichtigt. Ein wesentlicher Unterschied gegenüber dem bis 2009 angewandten Schätzverfahren [3], bei dem das Krebsregister des Saarlands von vornherein als vollzählig angenommen wurde, ist die Erweiterung der Referenzregion um diejenigen Register, die für den Zeitraum 1999 bis 2008 folgende Einschlusskriterien erfüllten:

- flächendeckende Erfassung über den gesamten Zeitraum,

- Vollzähligkeit für Krebs gesamt im Mittel über 90\% (nach der bisherigen Methode) und über $80 \%$ für alle Einzeljahre,

- DCO-Anteil für alle bösartigen Neubildungen (ICD10: C00-C97 ohne C44) seit 1999 oder ab dem 6. Jahr seit Beginn der Erfassung im Mittel unter $15 \%$.

Diese Kriterien wurden außer für das Saarland in den Bundesländern Hamburg, Bremen, Sachsen und im Regierungsbezirk Münster (NRW) erfüllt. Im Gegensatz zur früheren Methode lässt sich jetzt auch die Vollzähligkeit der Register aus der Referenzregion anhand des Vergleichs mit den Erwartungswerten schätzen. Erwartungswerte werden so für 6 Altersgruppen (15-39, 40 $49,50-59,60-69,70-79,80+$ Jahre) jeweils für beide Geschlechter sowie derzeit 18 Diagnosen bzw. Diagnosegruppen (- Tab. 1) berechnet. Um zufallsbedingte Schwankungen auszugleichen, werden sowohl erwartete als auch beobachtete Werte mittels log-linearer PoissonRegressionsmodelle geglättet (• Abb. 1 und Formelanhang A) [4]. Bei sehr geringer Mortalität ( $<5$ Sterbefälle durchschnittlich pro Jahr in der jeweiligen Altersgruppe und Untersuchungsregion) wird statt des M/I-Index ausnahmsweise die Inzidenz der Referenzregion für die Berechnung der Erwartungswerte herangezogen. Der geschätzte Erfassungsgrad in den einzelnen Bundesländern für „Krebs gesamt“ (C00-C97 ohne C44) wird schließlich über die Summation der beobachteten und erwarteten Werte für alle Einzelschätzungen berechnet. Anders als in den anderen Bundesländern erfolgt für Nordrhein-Westfalen aufgrund des regional unterschiedlichen
Standes der epidemiologischen Krebsregistrierung zurzeit noch eine Aufteilung in 3 Regionen: Regierungsbezirk Münster, übrige Region Westfalen-Lippe (Regierungsbezirke Detmold und Arnsberg) sowie Nordrhein (Regierungsbezirke Düsseldorf und Köln).

Die sich aus den log-linearen Modellen ergebenden Werte ermöglichen durch Verwendung von Bootstrap-Methoden erstmals auch die Bestimmung von Vertrauensbereichen für den geschätzten Erfassungsgrad. Bootstrapping findet besonders dann Verwendung, wenn die theoretische Verteilung einer interessierenden Statistik nicht bekannt ist bzw. nicht abgeleitet werden kann. In diesem Fall ist der geschätzte Erfassungsgrad das Ergebnis der Verknüpfung unterschiedlicher Modelle, sodass die Berechnung von Kovarianzen nicht möglich ist. Aus den gegebenen Einzelwerten werden durch wiederholtes Ziehen mit Zurücklegen Bootstrap-Stichproben generiert. Über die Varianz der daraus entstehenden Schätzwerte für den Erfassungsgrad erfolgt bei Vorliegen einer annähernden Normalverteilung die Berechnung eines approximativen $95 \%$-Vertrauensintervalls.

\section{Inzidenz: Schätzung der bundesweiten Krebshäufigkeit}

Das Prinzip der Schätzung der bundesweiten Inzidenz besteht darin, dass für „vollzählige“ Register die erfasste Inzidenz herangezogen wird, für „nicht-vollzählige" Register dagegen der Erwartungswert aus der Vollzähligkeitsschätzung. Als „vollzählig“" wird dabei ein geschätzter Erfassungsgrad von mindestens $90 \%$ definiert. Vereinfacht ausgedrückt wird damit eine etwa 10\%ige Abweichung des Verhältnisses von Mortalität zu Inzidenz im Vergleich zur Referenzregion noch als plausibel angesehen, eine größere Abweichung (nach oben) dagegen als Hinweis für mangelnde Vollzähligkeit eines Registers für die jeweilige Diagnosegruppe interpretiert.

Für „vollzählige" Register werden dabei entsprechend der Praxis in den Registern auch DCO-Fälle für die Ermittlung der Inzidenz berücksichtigt, das in der Regel unbekannte Diagnosejahr 
wird dabei durch das Sterbejahr ersetzt. Vor allem in der Aufbauphase eines Registers treten allerdings auch DCO-Fälle auf, die auf Neuerkrankungen vor dem Beginn der Registrierung zurückzuführen sind, was zu einer Überschätzung der Inzidenz führen würde. Daher wird in den ersten 5 Jahren eines Registers auch bei hoher Vollzähligkeit statt der eigenen DCO-Fälle der DCO-Anteil aus der Referenzregion den regulär erfassten Fällen hinzugerechnet.

Die bundesweiten Erkrankungszahlen für die einzelnen Lokalisationen und Diagnosejahre ergeben sich aus der Summation der Ergebnisse der jeweils „vollzähligen“ Register und der sich aus der Vollzähligkeitsschätzung ergebenden Erwartungswerte für die „nicht-vollzähligen“ Bundesländer. Auch diesen Erwartungswerten wird dabei der DCO-Anteil aus der Referenzregion hinzuaddiert. Für die Ergebnisse nach 5-Jahres-Altersgruppen (ab 15 Jahre) und Einzeldiagnosen (dreistellige ICD-10-Diagnosen) werden die Erwartungswerte unter der Annahme, dass die Vollzähligkeit sich innerhalb der definierten Diagnose- und Altersgruppen nicht unterscheidet, entsprechend aufgeteilt. Die Inzidenzen der Altersgruppen bis 15 Jahre werden vom Deutschen Kinderkrebsregister übernommen, da hier eine hohe bundesweite Vollzähligkeit angenommen werden kann [5].

\section{Überleben: Prüfung der Datenqualität}

Erste Voraussetzung für eine Nutzung von Krebsregisterdaten zur Berechnung von Überlebensraten ist das Vorhandensein ausreichend langer Zeitreihen. International ist für Krebserkrankungen die Publikation von 5-Jahres-Überlebensraten üblich. Für möglichst stabile Ergebnisse werden meist mehrere Diagnosejahre zusammengefasst. Daher können Überlebensraten in der Regel erst berichtet werden, wenn eine vollständige Nachverfolgung (Follow-up) bezüglich des Vitalstatus über 7 bis 8 Kalenderjahre hinweg erfolgt ist. Dieses Follow-up erfolgt in den Registern durch einen Abgleich mit allen Sterbefällen der Bezugsbevölkerung über Einwohnermeldeämter, Todesbescheinigungen und/oder statistische Landesäm-

Bundesgesundheitsbl 2014 · 57:13-21 DOI 10.1007/s00103-013-1878-z

(c) Springer-Verlag Berlin Heidelberg 2013

\section{K. Kraywinkel · B. Barnes · S. Dahm · J. Haberland · A. Nennecke · R. Stabenow Von regionalen Daten zu bundesweiten Aussagen. Die Methoden im Zentrum für Krebsregisterdaten}

\section{Zusammenfassung}

Trotz inzwischen erreichter Flächendeckung und deutlicher Verbesserung der Datenlage zur Epidemiologie von Krebserkrankungen in Deutschland werden bundesweite Ergebnisse zu Inzidenz, Überleben und Prävalenz vom Zentrum für Krebsregisterdaten (ZfKD) zurzeit geschätzt und nicht direkt aus allen vorliegenden Daten berechnet. Die hierzu verwendeten Verfahren beruhen dabei auf einer Schätzung der Vollzähligkeit der Erfassung von Neuerkrankungen bzw. auf einer Einschätzung der Datenqualität von Follow-upDaten. Die Vollzähligkeit der Erfassung wird dabei auf der Basis des M/I-Verfahrens geschätzt, welches von der Annahme eines in den Bundesländern weitgehend konstanten Verhältnisses von Mortalität und Inzi- denz einer Krebserkrankung bei gleichem Alter und Geschlecht ausgeht. Einschlusskriterien für die Aufnahme in den Datenpool zur Berechnung bundesweiter Überlebensraten sind ein DCO-Anteil unter $15 \%$ und plausible Überlebensraten für Pankreaskrebs und primär metastasierten Lungenkrebs. Die für das Jahr 2010 geschätzten ca. 477.300 jährlichen Krebserkrankungen beruhten bereits auf 429.900 erfassten Fällen (90\%), 10 Bundesländer trugen zuletzt zur Ermittlung bundesweiter Überlebensraten bei.

\section{Schlüsselwörter}

Krebserkrankungen · Register · Inzidenz · Überlebensraten · Deutschland

\section{Nationwide statements from regional data. Methods of The Center for Cancer Registry Data}

\section{Abstract}

Despite having achieved nationwide registry coverage in addition to substantial improvements in data on the epidemiology of cancer in Germany, the Centre for Cancer Registry Data continues to estimate national statistics on incidence, survival, and prevalence instead of calculating these directly from available data. The methods used for evaluations are based initially on estimates of registration completeness or, for survival analyses, an assessment of the quality of followup data. The completeness of incident case registration is estimated on the basis of the mortality/incidence procedure, which assumes a largely constant relationship between the mortality and incidence of a can- cer type among people of the same age and sex across federal states. Inclusion criteria for consideration of registry data in national survival analyses are less than $15 \%$ of death certificate only (DCO) cases and plausible survival for patients with pancreatic cancer or metastatic lung cancer. Of the 477,300 incident cancer cases estimated for 2010, 429,900 were reported by the cancer registries $(90 \%)$, and ten federal states contributed data to national survival estimates.

Keywords

Cancer - Registries · Incidence - Survival rate . Germany ter. Für die Prüfung der Qualität der so gewonnenen Daten zum Überleben der Patienten werden in internationalen Studien vor allem 2 Aspekte immer wieder zur Beurteilung herangezogen: der Anteil von DCO-Fällen und die Plausibilität von Überlebensraten bei Diagnosen mit sehr ungünstiger Prognose.

DCO-Fälle werden normalerweise aus Überlebenszeitanalysen ausgeschlossen, da das Diagnosedatum in Todesbescheinigungen meist nicht oder nur ungenau angegeben ist. Sie deuten allerdings auf ein potenziell schlechteres Überle- ben hin: In der Regel sind bei DCO-Fällen die Betroffenen an ihrer Krebserkrankung verstorben, was heute insgesamt nur noch auf knapp die Hälfte aller Krebspatienten zutrifft. Auch steigt die Wahrscheinlichkeit der Erfassung im Krebsregister bei längeren Überlebenszeiten $u$. a. durch die höhere Anzahl von Arztkontakten. Ein hoher Anteil ausgeschlossener DCO-Fälle kann demnach zu einer relevanten Überschätzung der Überlebensraten führen [6]. Für die Schätzung bundesweiter Überlebensraten durch das ZfKD werden daher nur diejenigen Register 


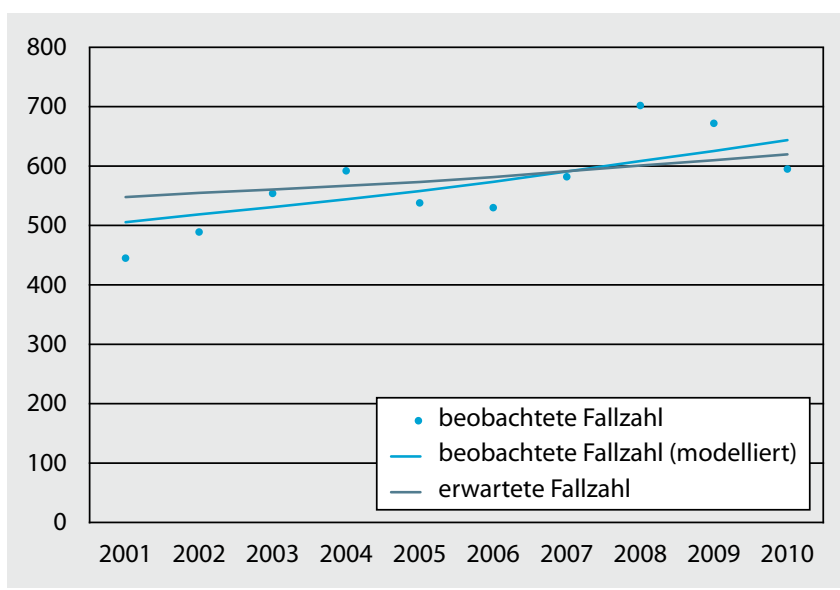

Abb. $1 \Delta$ Beobachtete und erwartete Anzahl jährlicher Neuerkrankungen Darmkrebs (C17-C21 und C26), Männer ab 15 Jahre, Krebsregister Hamburg, für die Jahre 2001-2010. Der geschätzte Erfassungsgrad für die einzelnen Diagnosejahre ergibt sich aus dem Quotient der Werte für die modellierte beobachtete Fallzahl geteilt durch die erwartete Fallzahl [für 2010: 103,8\% (95\%-Kl: 96,8-110,8\%)]

eingeschlossen, deren DCO-Anteil über den betrachteten Zeitraum für alle bösartigen Erkrankungen außer dem nichtmelanotischen Hautkrebs (C00-C97 ohne C44) unter 15\% lag.

Vergleichsweise hohe Überlebensraten bei Krebsdiagnosen mit ungünstiger Prognose, wie z. B. dem Pankreaskarzinom, weisen dagegen auf eine unvollständige Erfassung von Todesfällen im Register hin. Ein fehlerhafter bzw. unvollständiger Mortalitätsabgleich oder die mangelnde Erfassung von Wegzügen an Krebs erkrankter Personen aus dem Registergebiet kann dazu führen, dass Todesfälle im Register verpasst werden. Diese bleiben in der Regel langfristig unentdeckt und führen zu einem scheinbar besseren Überleben in der betroffenen Region. Ein Fehler dieser Art macht sich vor allem bei Diagnosen mit schlechter Prognose bemerkbar: Wenn z. B. nur $1 \%$ aller Todesfälle vom Register nicht erfasst werden, steigen die relativen 5-Jahres-Überlebensraten von Speiseröhrenoder Bauchspeicheldrüsenkrebs um etwa 1,3 Prozentpunkte, für Brustkrebs dagegen nur um 0,3 Prozentpunkte [7]. Die relativen 5-Jahres-Überlebensraten von Patienten mit Pankreaskarzinom und bei Diagnosestellung bereits fernmetastasiertem Lungenkrebs haben sich über lange Zeit nicht substanziell verbessert und liegen z. B. nach aktuellen Daten des USamerikanischen SEER-Programms bei et- wa 6 bzw. 4\% [8]. Daher wurde als zweites Kriterium ein Grenzwert von 7,5\% für das relative 5-Jahres-Überleben festgelegt, der für beide genannten Diagnosen (im Mittel) nicht überschritten werden soll. In Zweifelsfällen können weitere Kriterien (Stabilität der Ergebnisse über die Zeit, Überlebensraten bei weiteren Diagnosen mit schlechter Prognose) herangezogen werden. Für die für die Jahre 2009 und 2010 vom ZfKD berechneten Überlebensraten erfüllten insgesamt 10 Regionen (Hamburg, Niedersachsen, Bremen, Saarland, Brandenburg, Mecklenburg-Vorpommern, Thüringen, Sachsen, Rheinland-Pfalz und der Regierungsbezirk Münster) beide Kriterien und wurden damit in die Schätzung der bundesweiten Überlebensraten aufgenommen [9]. Daneben hat die Gesellschaft der epidemiologischen Krebsregister in Deutschland (GEKID) gemeinsam mit dem ZfKD Berichtstandards definiert, die die Vergleichbarkeit der für einzelne Register publizierten Ergebnisse erleichtern sollen [9].

\section{Überleben:}

\section{Berechnungsmethoden und Darstellung der Ergebnisse}

Die internationale Literatur bietet verschiedene Methoden zur Berechnung von Überlebenszeiten. Grundsätzlich werden Kohorten- und Periodenanalyse sowie absolute (auch: beobachtete) und relative Überlebensraten unterschieden. Die Angabe der absoluten Überlebensrate nach der Kohortenmethode ist die anschaulichste Darstellung der Überlebenschancen: Sie weist den Anteil derjenigen Krebspatienten eines definierten Diagnosezeitraums aus, die nach Ablauf einer bestimmten Zeit, z. B. 5 Jahre nach Diagnosestellung, noch leben. Rechnerisch werden hierbei bedingte Überlebenswahrscheinlichkeiten für das erste bis fünfte Jahr nach Diagnose berechnet und anschließend zur Ermittlung der Überlebensrate miteinander multipliziert. Die Aussagekraft des „absoluten“ Überlebens ist allerdings begrenzt, da auch Sterbefälle, die nicht im Zusammenhang mit der Krebserkrankung stehen, die Ergebnisse beeinflussen. Das "relative“ Überleben setzt daher das beobachtete Überleben ins Verhältnis zur allgemeinen Sterblichkeit von Personen gleichen Geschlecht und Alters, welches sich aus amtlichen Sterbetafeln entnehmen lässt. Dieses Vorgehen erlaubt vergleichbare Aussagen zum Einfluss der Krebserkrankung auf das Überleben von Patientenkollektiven unabhängig von einer Erfassung der Todesursachen und ist damit ein geeigneter Schätzer des krebsspezifischen Überlebens. Ein etwa für Hodenkrebspatienten inzwischen annähernd erreichtes relatives 5-Jahres-Überleben von 100\% bedeutet demnach, dass genauso viele $\mathrm{Pa}$ tienten 5 Jahre nach Diagnose dieser Erkrankung noch leben, wie anhand ihrer Alters- und Geschlechtsverteilung ohne Krebserkrankung zu erwarten wäre. Um, vor allem bei längerer Beobachtungszeit, Verzerrungen durch selektives Überleben der Jüngeren zu verhindern, werden für wissenschaftliche Publikationen und internationale Vergleiche spezielle Methoden zur Altersstandardisierung empfohlen $[11,12]$.

Die Periodenmethode wurde entwickelt, um die Prognose der in jüngster Zeit erkrankten Patienten besser zu schätzen bzw. Fortschritte in der onkologischen Therapie früher abbilden zu können. Analog der demografischen Berechnung der Lebenserwartung wird hier die Information zum Überleben aus einer möglichst aktuellen Kalenderperiode mit vollständig abgeschlossenem Mor- 

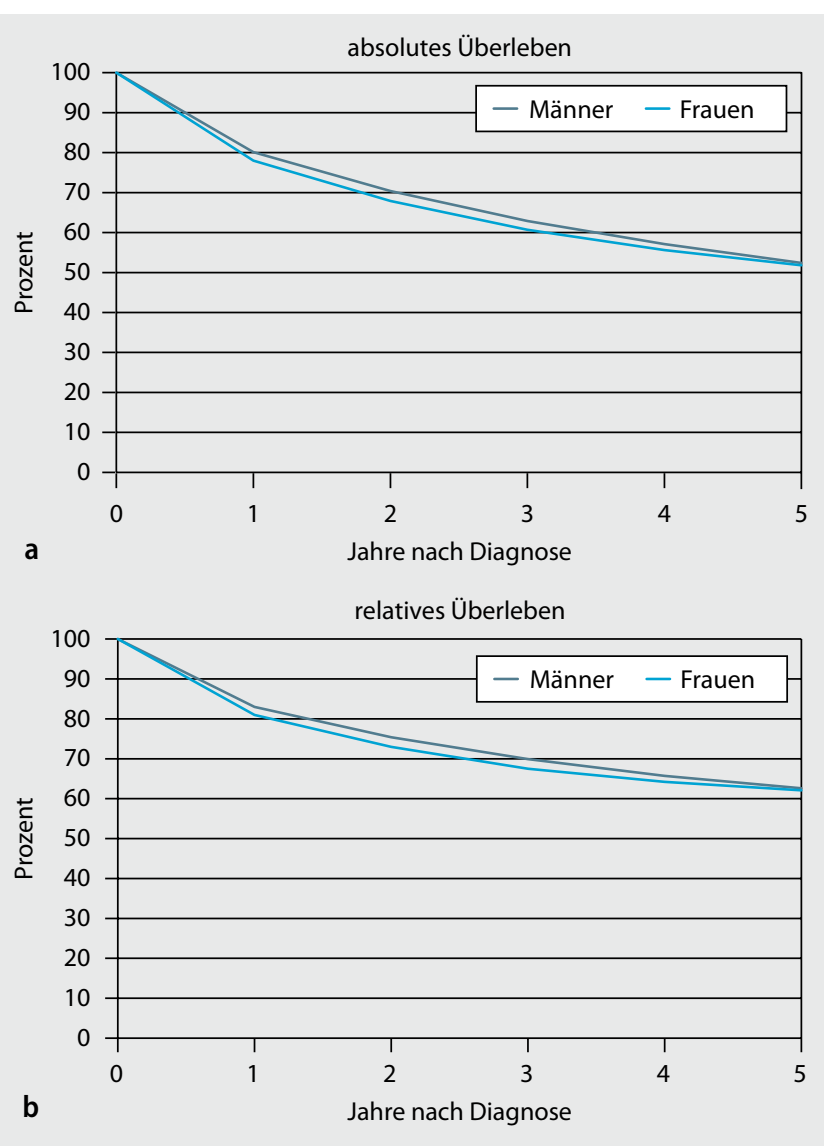

Abb. $2 \triangle$ Absolute (a) und relative (b) Überlebensraten bei Darmkrebs (C18-C21), bis 5 Jahre nach Diagnose, Deutschland, Periode 2009-2010 [8]
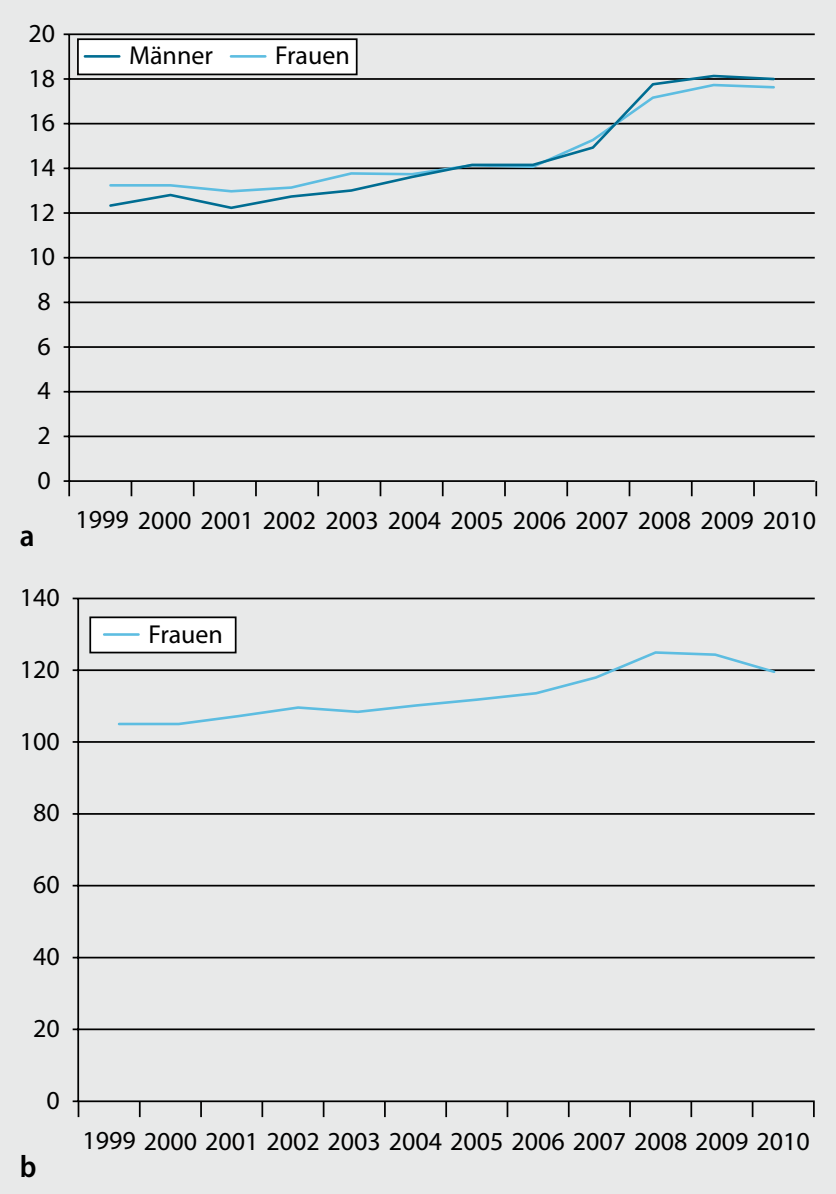

Abb. $3 \Delta$ a Altersstandardisierte Inzidenzrate für das maligne Melanom der Haut (C43), Männer und Frauen, Deutschland 1999 bis 2010, nach Europastandard (Schätzung ZfKD). b Altersstandardisierte Inzidenzrate Brustkrebs (C50) Frauen, Deutschland 1999 bis 2010, nach Europastandard (Schätzung ZfKD) talitätsabgleich herangezogen. Für die Periode 2009-2011 gehen für das Überleben im ersten Jahr nach Diagnose zwischen 2008 und 2011 erkrankte Personen ein, für das Überleben im zweiten Jahr solche aus den Diagnosejahren 2007 bis 2010, und schließlich für das fünfte Jahr Personen, die zwischen 2004 und 2007 erkrankt sind. Aus den bedingten Überlebenswahrscheinlichkeiten für das erste bis fünfte Jahr werden dann wie bei der Kohortenmethode durch Multiplikation 5-Jahres-Überlebensraten berechnet. Der wesentliche Unterschied besteht darin, dass bei der Periodenmethode auch $\mathrm{Pa}$ tienten, die erst in jüngerer Zeit diagnostiziert wurden, zu den Ergebnissen beitragen. An historischen Daten konnte gezeigt werden, dass die Ergebnisse der Periodenmethode den Überlebensraten der in der entsprechenden Periode tatsäch- lich Erkrankten in der Regel sehr nahe kommen [13].

Für die Routineberichterstattung in „Krebs in Deutschland“ berechnet das ZfKD alle 2 Jahre absolute und relative Überlebensraten nach der Periodenmethode ohne Altersstandardisierung (- Abb. 2). Da international verschiedene Berechnungsweisen nebeneinander existieren, werden die Ergebnisse zur besseren Vergleichbarkeit auf der Homepage des ZfKD auch altersstandardisiert und nach der Kohortenmethode berechnet zur Verfügung gestellt. Mittelfristig ist die Veröffentlichung von 10-Jahres-Überlebensraten geplant, für deren Berechnung momentan noch zu wenige Register die notwendigen Zeitreihen aufweisen. Die Berechnung der Überlebensraten erfolgt mit dem Paket „periodR“ für die Statistiksoftware R [14].

\section{Schätzung der Prävalenz}

Bei Krebserkrankungen wird in der Regel die sog. Periodenprävalenz (engl. „limited duration-“ oder „partial prevalence“) betrachtet. So bezeichnet die 5-Jahres-Prävalenz die Zahl, der zu einem gegebenen Zeitpunkt noch lebenden Patienten, die innerhalb der letzten 5 Jahre an Krebs erkrankt sind. Diese Zahl bietet ein orientierendes Maß für die Belastung des Gesundheitssystems über die akute Erkrankungsphase hinaus, auch wenn die Dauer des Versorgungsbedarfs im Einzelnen stark nach Art und Verlauf der Krebserkrankung variiert. In vollzähligen Registern mit ausreichend langen Zeitreihen kann die Periodenprävalenz direkt durch „Auszählung“ bestimmt werden. Im ZfKD wird die Prävalenz nach der Methode von Pi- 
sani unter Nutzung der oben beschriebenen Schätzungen für Inzidenz- und Überlebensraten näherungsweise berechnet. Unter der Annahme einer innerhalb eines Kalenderjahres konstanten Inzidenz wird für die 1-Jahres-Prävalenz am Ende des Jahres 2010 die geschätzte Zahl der Neuerkrankungen aus 2010 mit der bundesweiten, absoluten 0,5-Jahres-Überlebensrate aus dem gleichen Jahr multipliziert (nach Altersgruppe und Geschlecht). Für die 2-Jahres-Prävalenz wird das Produkt aus den Neuerkrankungen des Jahres 2009 mit der 1,5 Jahres-Überlebensrate hinzuaddiert, entsprechend werden alle weiteren Ergebnisse bis zur 5-Jahres-Prävalenz berechnet (s. Formelanhang B) [15]. Geplant sind Weiterentwicklungen der Methode zur Abschätzung der Lebenszeitprävalenz bzw. der Anzahl der Langzeitüberlebenden.

\section{Diskussion}

\section{Schätzung der Vollzähligkeit der Erfassung in den epidemiologischen Krebs- registern}

Es existieren international verschiedene, auf unterschiedlichen Annahmen beruhende Ansätze zur Schätzung der Vollzähligkeit von Krebsregistern [16, 17]. Einige von ihnen setzen ein möglichst exaktes Meldedatum (Flow-Methode) oder die Angaben verschiedener Meldequellen (Capture-Recapture-Verfahren) voraus und sind deshalb für die im ZfKD vorliegenden Daten nicht anwendbar $[18,19]$. Andere Verfahren sind aufgrund komplexer Modellierungen erst für längere Zeitreihen geeignet [20]. Auch die Beurteilung der Vollzähligkeit über den DCO-Anteil, wie sie von der „International Agency for Research on Cancer" (IARC) zur Aufnahme in internationale Datensammlungen verwendet wird [21], ist frühestens etwa 5 Jahre nach Gründung eines Registers aussagekräftig. AuBerdem existieren zwischen den Bundesländern Unterschiede, inwieweit nur das "Grundleiden“ oder auch „sonstige Erkrankungen" aus einer Todesbescheinigung zur Definition eines DCO-Falles verwendet werden, sodass keine ex- akte Vergleichbarkeit der DCO-Anteile gegeben ist. Für die föderal geprägte Situation in Deutschland mit nach wie vor einigen im Aufbau befindlichen Registern scheint die M/I-Methode daher am besten zur Vollzähligkeitsschätzung geeignet, zumal die Annahme eines innerhalb Deutschlands weitgehend konstanten M/I-Index für die meisten Krebsarten plausibel sein dürfte. Diese grundlegende Annahme kann vor allem dann verletzt sein, wenn Früherkennungsmethoden existieren, die regional unterschiedlich stark wahrgenommen werden (z. B. in der Anlaufphase des Mammographiescreenings), oder wenn sich unterschiedliche Subtypen einer Erkrankung bzw. Erkrankungsgruppe mit unterschiedlicher Prognose regional heterogen verteilen (möglich beim Schilddrüsenkarzinom oder der Gruppe der Kopf-HalsTumoren). Auch die Annahme einer vollzähligen Registrierung in den 5 Referenzregistern dürfte nicht gleichbleibend über alle Lokalisationen zu 100\% erfüllt sein. Schließlich ist die Beurteilung der Vollzähligkeit anhand des M/IVerhältnisses (bzw. ersatzweise anhand der beobachteten Inzidenz) bei Krebsarten mit geringer Mortalität gerade in kleineren Registern aufgrund geringer Fallzahlen schwierig und erzeugt instabile Ergebnisse bzw. weite Konfidenzintervalle. Es zeichnet sich jedoch ab, dass in den nächsten Jahren weitere Register die oben genannten Kriterien zur Aufnahme in die „Referenzregion" erfüllen werden, was die Stabilität der Schätzungen von Jahr zu Jahr weiter erhöhen dürfte.

\section{Schätzung der \\ bundesweiten Inzidenz}

Auch für die Schätzung nationaler Inzidenzraten bei nicht flächendeckender Krebsregistrierung werden international verschiedene Methoden genutzt. Länder mit annähernd flächendeckender Krebsregistrierung wie die Schweiz verwenden meist einfache Hochrechnungen anhand beobachteter altersspezifischer Inzidenzraten und den nationalen Bevölkerungsdaten [22]. Dagegen werden z. B. in Frankreich mit eher geringer Abdeckung durch kleinere, aber bereits länger etablierte Register komplexere Mo- dellierungsverfahren eingesetzt [23]. Mit der im ZfKD entwickelten Methode wurde für Deutschland ein Kompromiss gewählt, der der inzwischen deutlich verbreiterten Datenlage Rechnung trägt und gleichzeitig berücksichtigt, dass bevölkerungsreiche Bundesländer wie $\mathrm{Ba}$ den-Württemberg oder Hessen zum jetzigen Zeitpunkt noch keine vollzählige Registrierung aufweisen. Die gewählte Methode ermöglicht eine automatische Anpassung an eine zunehmende Vollzähligkeit dieser noch im Aufbau befindlichen Register und gewährleistet damit quasi einen fließenden Übergang vom „Schätzen“ zum „Zählen“. Durch Nutzung der Erwartungswerte aus der Vollzähligkeitsschätzung für zurückliegende Jahre ab 1999 konnten die zeitlichen Entwicklungen der Inzidenzraten über diesen Zeitraum mit konsistenter Methodik abgebildet werden. Damit wurde eine Beurteilung der Effekte der Einführung des organisierten Mammographiescreenings bzw. der Erweiterung des Angebots zur Hautkrebsfrüherkennung (Ganzkörperinspektion durch Dermatologen oder Hausarzt) auf die bundesweiten Erkrankungsraten möglich. Beide Maßnahmen waren zunächst mit einem deutlichen Anstieg der Neuerkrankungsraten verbunden, der bei der Mammographie aufgrund der zeitlich versetzten Einführung in verschiedenen Regionen Deutschlands zwischen 2005 und 2009 weniger abrupt erfolgte als bei der Mitte 2008 in ganz Deutschland eingeführten Hautkrebsfrüherkennung (• Abb. 3a,b). Die insgesamt gute Übereinstimmung mit von der IARC auf der Basis älterer Daten vorhergesagten deutschlandweiten Inzidenzen im Rahmen des GLOBOCAN-Projektes sowie mit den Ergebnissen aus den Nachbarländern Deutschlands deutet darauf hin, dass die aktuellen Inzidenzraten nicht wesentlich über- oder unterschätzt sein dürften $[9,24]$.

Ein Nachteil der neuen Methode besteht darin, dass mit ihr zunächst keine retrospektiven Aussagen zu längerfristigen Entwicklung der Krebsinzidenzen in Deutschland möglich sind, obwohl für einige Regionen Deutschlands bereits seit mehreren Jahrzehnten neben den Mortalitätsstatistiken auch belastbare Zahlen zu den Neuerkrankungen zur Verfügung 
stehen. Hier gilt es, in den nächsten Jahren ein neues Verfahren zu entwickeln, das diese Lücke schließt.

\section{Schätzung bundesweiter Überlebensraten}

Die Schwierigkeit der Berechnung bundesweiter Überlebensraten liegt vor allem in der Beurteilung der Datenqualität. Es existieren keine international anerkannten Richtwerte, ab welchem Wert beispielsweise Überlebensraten nach Pankreaskarzinom nicht mehr plausibel sind oder welcher Anteil auszuschließender DCO-Fälle noch akzeptiert werden kann.

Grundsätzlich würden die oben beschriebenen Fehlermöglichkeiten, also das Verpassen von Todesfällen im Register oder die selektive Verzerrung durch Ausschließen von DCO-Fällen zu einer Überschätzung der Überlebensraten führen. Daraus ist aber nicht unbedingt $\mathrm{zu}$ folgern, dass die Bundesländer mit den niedrigsten Überlebensraten automatisch die beste Datenqualität aufweisen: Einerseits wären gewisse Unterschiede in den Überlebensraten innerhalb Deutschlands durchaus als plausibel anzusehen, andererseits können hier auch Unterschiede in der Erfassung eine Rolle spielen. So werden in einigen Registern ursprünglich über die Todesbescheinigung bekannt gewordene Fälle (Death Certificate Notified - DCN-Fälle) durch Nachfragen bei dem den Leichenschauschein ausstellenden Arzt zurückverfolgt (Trace-back) [25]. Hierdurch werden eher solche Fälle aufgeklärt, deren Diagnosedatum kürzer zurückliegt und die damit eine geringere Überlebenszeit aufweisen. Auf diese Weise besteht grundsätzlich die Möglichkeit einer selektionsbedingten Unterschätzung des Überlebens für diejenigen Register bzw. Krebsarten, für die das Trace-back-Verfahren einen hohen Anteil der registrierten Fälle generiert [26].

Vergleiche mit den publizierten Ergebnissen des finnischen Krebsregisters mit ausgewiesen guter Datenqualität deuten jedoch eher darauf hin, dass die vom ZfKD für Deutschland publizierten Überlebensraten zumindest für Erkrankungen mit ungünstiger Prognose noch leicht überschätzt sind. Insgesamt ist die Vergleichbarkeit mit publizierten Ergeb- nissen aus Europa oder den USA jedoch durchaus gegeben $[8,27,28]$.

Es muss betont werden, dass die jetzige Situation bereits deutlich repräsentativere Aussagen zu den bundesweiten Überlebensraten nach Krebserkrankungen zulässt als etwa noch vor 5 Jahren: Damals wurden sowohl von der Dachdokumentation Krebs als auch in internationalen Studien für Deutschland lediglich die Daten des Saarlandes herangezogen, was alleine schon aufgrund der Fallzahl die Aussagekraft für bestimmte Krebsarten einschränkte $[28,29]$. In der zurzeit laufenden fünften Auflage der EUROCARE-Studie, in der Überlebensraten nach Krebs aus fast allen europäischen Staaten analysiert werden, ist nun dagegen erstmals eine ganze Reihe deutscher Register vertreten [30].

Aufgrund der besonderen Rolle von Überlebensraten als Qualitätsindikatoren der onkologischen Versorgung und der beschriebenen Verzerrungsmöglichkeiten ist jedoch einerseits vor einem unkritischen Vergleich publizierter Einzelergebnisse (im Sinne eines "Ranking“) $\mathrm{zu}$ warnen, anderseits sollte eine weitere Verbesserung der Datenqualität gerade auch im Rahmen des Ausbaus der klinischen Krebsregistrierung angestrebt werden.

\section{Fazit}

Die epidemiologische Krebsregistrierung in Deutschland befindet sich auf dem Weg zu einer bundesweiten vollzähligen Erfassung von Krebserkrankungen, wie sie international bisher nur in sehr wenigen Ländern mit vergleichbarer Bevölkerungsgröße erreicht ist. Von den ca. 477.300 geschätzten Erkrankungsfällen im Jahr 2010 wurden bereits etwa $90 \%$ in den Registern erfasst. Seit 1999 hat sich dieser Anteil nahezu verdoppelt. Alleine durch den weiteren Aufbau des Registers in Baden-Württemberg ist zu erwarten, dass der bundesweite Erfassungsgrad weiter zunehmen wird. Dennoch bestehen in einigen Bundesländern teilweise strukturell bedingte Defizite in der Vollzähligkeit der Erfassung von Neuerkrankungen und/oder Sterbefällen. Der jetzt initiierte bundesweite Ausbau der klinischen Krebsregist- rierung sollte daher genutzt werden, um die bestehende Defizite auszugleichen, denn letztlich hängt die Nutzbarkeit sowohl klinischer als auch epidemiologischer Krebsregistrierung wesentlich von der Qualität der Daten ab.

Die derzeit im ZfKD verwandten Methoden zur Schätzung von Inzidenz und Überleben sind auf den hier beschriebenen aktuellen Stand der Krebsregistrierung in Deutschland abgestimmt. Es erfolgt zunächst eine standardisierte Prüfung der Registerdaten auf Vollzähligkeit bezüglich der Inzidenz bzw. auf Plausibilität der Ausgangsdaten für Überlebenszeitanalysen. Bei Erfüllung festgelegter Kriterien werden die Daten weitgehend unverändert zur Schätzung bundesweiter Ergebnisse verwendet. Eine weitere Verbesserung der Datenqualität wird es in Zukunft ermöglichen, diese Schätzmethoden nach und nach durch die Verwendung aller Daten für eine „einfache“ Zählung bzw. Berechnung von Inzidenz, Prävalenz und Überlebensraten zu ersetzen. Für Fragestellungen, die prinzipiell lange Zeitreihen voraussetzen (langfristige Inzidenztrends, Langzeitüberleben, Lebenszeitprävalenz), werden bundesweite Aussagen allerdings auch weiterhin zunächst nur unter Verwendung komplexer Schätzmethoden getroffen werden können, hierfür sind teilweise noch methodische Weiterentwicklungen notwendig.

\section{Korrespondenzadresse}

\section{Dr. K. Kraywinkel}

Zentrum für Krebsregisterdaten,

Robert Koch-Institut

General-Pape-Str. 62-66,

12101 Berlin

k.kraywinkel@rki.de

\section{Einhaltung ethischer Richtlinien}

Interessenkonflikt. K. Kraywinkel, B. Barnes, S. Dahm, J. Haberland, A. Nennecke und R. Stabenow geben an, dass kein Interessenkonflikt besteht.

Dieser Beitrag beinhaltet keine Studien an Menschen oder Tieren. 


\section{Literatur}

1. Bundeskrebsregisterdatengesetz (2009) In: Begleitgesetz zur zweiten Föderalismusreform vom 10. August 2009. Bundesgesetzblatt Jahrgang 2009 Teil I Nr. 53, S 2707-2708

2. Schön D, Bertz J, Görsch B et al (2004) Die Dachdokumentation Krebs - Eine Surveillance-Einrichtung der Krebsregistrierung in Deutschland. Bundesgesundheitsbl Gesundheitsforsch Gesundheitsschutz 47:429-436

3. Haberland J, Schön D, Bertz J, Görsch B (2003) Vollzähligkeitsschätzungen von Krebsregisterdaten in Deutschland. Bundesgesundheitsbl Gesundheitsforsch Gesundheitsschutz 46:770-774

4. Hakulinen T, Dyba T (1994) Precision of incidence predictions based on Poisson distributed observations. Stat Med 13(15):1513-1523

5. Kaatsch P (2004) Das Deutsche Kinderkrebsregister im Umfeld günstiger Rahmenbedingungen. Bundesgesundheitsbl Gesundheitsforsch Gesundheitsschutz 47(5):437-443

6. Brenner H, Holleczek B (2011) Deriving valid population-based cancer survival estimates in the presence of nonnegligible proportions of cancers notified by death certificates only. Cancer Epidemiol Biomarkers Prev 20(12):2480-2486

7. Brenner H, Hakulinen T (2009) Implications of incomplete registration of deaths on long-term survival estimates from population-based cancer registries. Int J Cancer 125(2):432-437

8. Surveillance, Epidemiology, and End Results (SEER) Program. http://seer.cancer.gov

9. Robert Koch-Institut, Die Gesellschaft der epidemiologischen Krebsregister in Deutschland e.V. (Hrsg) (2013) Krebs in Deutschland 2009/2010. Robert Koch-Institut, Berlin

10. Nennecke A, Barnes B, Brenner H et al (2013) Datenqualität oder Unterschiede in der onkologischen Versorgung? - Berichtsstandards für Überlebenszeitanalysen mit Krebsregisterdaten. Gesundheitswesen 75(2):94-98

11. Verdecchia A, Capocaccia R, Santaquilani M, Hakulinen T (1999) Methods of survival data analysis and presentation issues survival of cancer patients in Europe: the EUROCARE-2 Study. IARC Scientific Publication No. 151, International Agency for Research on Cancer, Lyon, S 41-45

12. Brenner $\mathrm{H}$, Hakulinen $\mathrm{T}$ (2005) Age adjustment of cancer survival rates: methods, point estimates and standard errors. Br J Cancer 93(3):372-375

13. Brenner H, Gefeller O, Hakulinen T (2004) Period analysis for "up-to-date" cancer survival data: theory, empirical evaluation, computational realisation and applications. Eur J Cancer 40(3):326335

14. Holleczek B, Gondos A, Brenner H (2009) periodR - an $R$ package to calculate long-term cancer survival estimates using period analysis. Methods Inf Med 48(2):123-128

15. Pisani P, Bray F, Parkin DM (2002) Estimates of the world-wide prevalence of cancer for 25 sites in the adult population. Int J Cancer 97(1):72-81

16. Parkin DM, Bray F (2009) Evaluation of data quality in the cancer registry: principles and methods Part II: Completeness. Eur J Cancer 45(5):756-764

17. Schmidtmann I, Blettner M (2009) How do cancer registries in europe estimate completeness of registration? Methods Inf Med 48(3):267-271

18. Bullard J, Coleman MP, Robinson D et al (2000) Completeness of cancer registration: a new method for routine use. Br J Cancer 82:1111-1116
19. Robles SC, Marrett LD, Clarke EA, Risch HA (1988) An application of capture-recapture methods to the estimation of completeness of cancer registration. J Clin Epidemiol 41:495-501

20. Capocaccia R, Buzzoni C, Grande E et al (2007) Estimated and observed cancer incidence in Italy: a validation study. Tumori 93(4):387-391

21. Curado MP, Edwards B, Shin HR et al (Hrsg) (2007) Cancer incidence in five continents, Vol. IX. IARC Scientific Publications No. 160, International Agency for Research on Cancer, Lyon

22. Bundesamt für Statistik, National Institute for Cancer Epidemiology and Registration, Schweizer Kinderkrebsregister (Hrsg) (2011) Krebs in der Schweiz. Stand und Entwicklung von 1983 bis 2007. Office fédéral de la statistique, Neuchâtel

23. Belot A, Grosclaude P, Bossard N et al (2008) Cancer incidence and mortality in France over the period 1980-2005. Rev Epidemiol Sante Publique 56(3):159-175

24. Ferlay J, Shin HR, Bray F et al (2010) Estimates of worldwide burden of cancer in 2008: GLOBOCAN 2008. Int J Cancer 127(12):2893-2917

25. Nennecke A, Brenner H, Eberle A et al (2010) Überlebenschancen von Krebspatienten in Deutschland - auf dem Weg zu repräsentativen, vergleichbaren Aussagen. Gesundheitswesen 72(10):692-699

26. Holleczek B, Brenner H (2012) Reduction of population-based cancer survival estimates by trace back of death certificate notifications: an empirical illustration. Eur J Cancer 48(6):797-804

27. Finnish Cancer Registry. http://www.cancer.fi/syoparekisteri/en/statistics/newest-survival-ratios

28. Verdecchia A, Francisci S, Brenner $\mathrm{H}$ et al (2007) Recent cancer survival in Europe: a 2000-02 period analysis of EUROCARE-4 data. Lancet Oncol 8:784-796

29. Coleman MP, Quaresma M, Berrino F et al (2008) Cancer survival in five continents: a worldwide population-based study (CONCORD). Lancet Oncol 9(8):730-756

30. Istituto Superiore di Sanità, Rom. EUROCARE-5, geographical coverage. http://www.eurocare.it/ Eurocare5/ResultsEU5/tabid/90/Default.aspx 


\section{Formelanhang}

A. Vorgehen bei der Vollzähligkeitsschätzung im ZfKD

\section{Mortalitätsanalyse für}

\section{Referenzregion $\mathbf{R}$}

$\operatorname{InE}\left(M_{i t}^{R}\right)=\operatorname{In} P_{i t}^{R}+a_{0 i}+a_{1 i} t \rightarrow \hat{M}_{i t}^{R}$

\section{Inzidenzanalyse für} Referenzregion $\mathbf{R}$

$\operatorname{In} \mathrm{E}\left(I_{i t}^{R}\right)=\operatorname{In} P_{i t}^{R}+\beta_{0 i}+\beta_{1 i} t \rightarrow \hat{I}_{i t}^{R}$

\section{Mortalitätsanalyse für} Untersuchungsregion $\mathrm{U}$

$\operatorname{In} \mathrm{E}\left(M_{i t}^{U}\right)=\operatorname{In} P_{i t}^{U}+\gamma_{0 i}+\gamma_{1 i} t \rightarrow \hat{M}_{i t}^{U}$

\section{Erwartete Inzidenz in der} Untersuchungsregion $\left(\mathrm{U}_{\mathrm{erw}}\right)$

$I_{i t}^{U e r y}=\hat{M}_{i t}^{U} \frac{\hat{I}_{i t}^{R}}{\hat{M}_{i t}^{R}}$

\section{Inzidenzanalyse}

(Untersuchungsregion U)

$\operatorname{InE}\left(I_{i t}^{U}\right)=\operatorname{In} P_{i t}^{U}+\delta_{0 i}+\delta_{1 i} t \rightarrow \hat{I}_{i t}^{U}$

\section{Erfassungsgrad}

$$
\frac{\hat{I}_{\cdot T}^{U}}{I_{\cdot T}^{U e r w}}
$$

ln: natürlicher Logarithmus E: Erwartungswert i: Alter t: Jahre M: Verstorbene I: Erkrankte P: Bevölkerung $\alpha, \beta, \gamma, \delta$ : Koeffizienten

\section{B. Prävalenzschätzung nach Pisani}

Die $n$-Jahres-Prävalenz von Patienten im Alter $k$ am Ende des Kalenderjahres $j$ wird geschätzt durch:

$\operatorname{Pr} e(n, k, j)$

$=\sum_{t=0}^{\min (n-1, k)} \operatorname{In} c_{k-t, j-t} \cdot S_{k-t, j-t}(t+0.5)$

$I n c_{\mathrm{k}-\mathrm{t}, \mathrm{j}-\mathrm{t}}=$ jährliche Anzahl von Neuerkrankungen im Alter $k-t$ aus dem Jahr $\mathrm{j}-\mathrm{t}$

$\mathrm{S}_{\downarrow}(\mathrm{k}-\mathrm{t}, \mathrm{j}-\mathrm{t})(\mathrm{t}+0.5)$

$=$ Überlebenswahrscheinlichkeit für $\mathrm{t}+0,5$ Jahre im Alter $\mathrm{k}-\mathrm{t}$ für Neuerkrankungen aus dem Jahr $\mathrm{j}-\mathrm{t}$ 